\title{
EXTENDING UNIFORMLY CONTINUOUS PSEUDO- ULTRAMETRICS AND UNIFORM RETRACTS
}

\author{
ROBERT L. ELLIS ${ }^{1}$
}

\begin{abstract}
It is first proved that any uniformly continuous pseudo-ultrametric on a subspace of a non-Archimedean uniform space $X$ has a uniformly continuous extension to $X$ (which preserves total boundedness or separability). Then it is proved that every complete subspace of an ultrametrizable space $X$ is a uniform retract of $X$. This has consequences concerning the extension of uniformly continuous functions.
\end{abstract}

Isbell [6, Lemma 1.4] proved that every bounded uniformly continuous pseudometric $\rho$ on a subspace of a uniform space $X$ has an extension to a bounded uniformly continuous pseudometric on $X$. In this paper we consider the same problem when $X$ is a non-Archimedean uniform space (see [7] for terminology) and $\rho$ is a pseudoultrametric, i.e., $\rho$ satisfies the strong triangle inequality $\rho(x, y)$ $\leqq \max \{\rho(x, z), \rho(z, y)\}$. In this connection we mention that the topology of any Hausdorff space of small inductive dimension zero is defined by a non-Archimedean uniform structure. It is proved that, even if $\rho$ is not bounded, it has an extension to a uniformly continuous pseudo-ultrametric on $X$. There is also interest [1], [2], [5] in extending pseudometrics which are either totally bounded or separable, and we prove that if $\rho$ has one of these properties, then it has a uniformly continuous extension which has the same property.

The above result is obtained as a simple corollary of a theorem which states that if $\rho$ is a uniformly continuous pseudo-ultrametric on a subset $S$ of a non-Archimedean uniform space $X$, then there is a uniformly continuous extension $\Phi: X \rightarrow S^{*}$ of the canonical map $\phi: S$ $\rightarrow S^{*}$ of $S$ into its completion $S^{*}$ for $\rho$. We say that a subset $S$ of a uniform space $X$ is a uniform retract of $X$ if there is a uniformly continuous function from $X$ into $S$ which extends the identity function on $S$. Another corollary is that any complete subset of a pseudoultrametric space $X$ is a uniform retract of $X$. This has implications concerning the extension of uniformly continuous functions.

Received by the editors October 28, 1970.

AMS 1970 subject classifications. Primary 54C20, 54C15. tract.

Key words and phrases. Non-Archimedean uniform space, ultrametric, uniform re-

${ }^{1}$ This work was supported by a Faculty Research Award from the University of Maryland. 
Lemma. Let $S$ be a subset of a non-Archimedean uniform space $X$. If $V$ is a non-Archimedean entourage on $S$, there is a non-Archimedean entourage $V^{\prime}$ on $X$ such that $V^{\prime} \cap(S \times S)=V$.

Proof. Let $W$ be an entourage on $X$ such that $W \cap(S \times S)=V$ and let $T$ be a non-Archimedean entourage on $X$ contained in $W$. Let

$$
V^{\prime}=\bigcup_{n=1}^{\infty}(T \cup V)^{n} \text {. }
$$

Then $V^{\prime}$ is clearly a non-Archimedean entourage on $X$. We will prove that $V^{\prime} \cap(S \times S)=V$ by showing by induction on $n$ that any set of the form $A_{1} \circ A_{2} \circ \cdots \circ A_{n}$, where each $A_{i}$ is either $T$ or $V_{i}$ intersects $S \times S$ inside $V$. This is clear if either $n=1$ or each $A_{i}=T$. If $n>1, A_{i_{0}}=V$, and $\left(s_{1}, s_{n+1}\right) \in A_{1} \circ A_{2} \circ \cdots \circ A_{n} \cap(S \times S)$, then $\left(s_{1}, s_{n+1}\right)=\left(s_{1}, x_{2}\right) \circ\left(x_{2}, x_{3}\right) \circ \cdots \circ\left(x_{n}, s_{n+1}\right)$, where $\left(x_{i}, x_{i+1}\right) \in A$, $\left(x_{1}=s_{1}, x_{n+1}=s_{n+1}\right)$. Since $A_{i_{0}}=V, x_{i_{0}}$ and $x_{i_{0}+1} \in S$. By the inductive hypothesis, if $i_{0} \neq n$, then $\left(s_{1}, x_{2}\right) \circ\left(x_{2}, x_{3}\right) \circ \cdots \circ\left(x_{i_{0}}, x_{i_{0}+1}\right)$ and $\left(x_{i_{0}+1}, x_{i_{0}+2}\right) \circ \cdots \circ\left(x_{n}, s_{n+1}\right)$ belong to $V$ and so $\left(s_{1}, s_{n+1}\right) \in V^{2}=V$. Similarly, if $i_{0}=n$, then $\left(s_{1}, x_{2}\right) \circ\left(x_{2}, x_{3}\right) \circ \cdots \circ\left(x_{n-1}, x_{n}\right)$ and $\left(x_{n}, s_{n+1}\right) \in V$ and so $\left(s_{1}, s_{n+1}\right) \in V^{2}=V$.

THEOREM 1. Let $\rho$ be a uniformly continuous pseudo-ultrametric on a subset $S$ of a non-Archimedean uniform space $X$. Then there is a uniformly continuous function $\Phi: X \rightarrow S^{*}$ which extends the canonical map $\phi: S \rightarrow S^{*}$, where $\left(S^{*}, \rho^{*}\right)$ is the completion of $(S, \rho)$.

PROOF. For each in teger $n \geqq 1,\{(x, y) \mid \rho(x, y)<1 / n\}$ is a symmetric non-Archimedean entourage on $S$. By the lemma there is a sequence $\left\{V_{n} \mid n \geqq 1\right\}$ of symmetric non-Archimedean entourages on $X$ such that $V_{1}=X \times X, V_{n+1} \subseteq V_{n}$, and for $n>1, V_{n} \cap(S \times S)=\{(x, y)$ $\mid p(x, y)<1 / n\}$. Then $v_{n}=\left\{V_{n}(s) \mid s \in S\right\}$ is a partition of $V_{n}(S)$ [4, Lemma 5.3]. Let $\left\{s_{i} \mid i \in I_{n}\right\} \subseteq S$ be a complete set of representatives of $\vartheta_{n}$. Define a function $N$ from $X$ into $N \cup\{\infty\}$ by

$$
N(x)=\sup \left\{n \mid x \in V_{n}(S)\right\} .
$$

If $n$ is an integer and $1 \leqq n \leqq N(x)$ let $i(n, x) \in I_{n}$ be such that $V_{n}(x)$ $=V_{n}\left(s_{i(n, x)}\right)$. For each integer $n \geqq 1$ define a function $f_{n}: X \rightarrow S$ by

$$
\begin{aligned}
f_{n}(x) & =s_{i(N(x), x)}, & & \text { if } N(x)<\infty, \\
& =s_{i(n, x)}, & & \text { if } N(x)=\infty .
\end{aligned}
$$

We first show that for all $x \in X,\left\{f_{n}(x) \mid n \geqq 1\right\}$ is a Cauchy sequence, which is trivial if $N(x)<\infty$. If $N(x)=\infty$ and $m \geqq n>1$, then 
$\left(f_{m}(x), f_{n}(x)\right)=\left(s_{i(m, x)}, x\right) \circ\left(x, s_{i(n, x)}\right) \in V_{m} \circ V_{n}=V_{n}$ and so $\rho\left(f_{m}(x), f_{n}(x)\right)<1 / n$. Define $\Phi: X \rightarrow S^{*}$ by letting $\Phi(x)$ be the equivalence class in $S^{*}$ of the Cauchy sequence $\left\{f_{n}(x) \mid n \geqq 1\right\}$. Then $\Phi$ extends $\phi$ because if $s \in S$, then $N(s)=\infty$ and for each $n \geqq 2$, $\left(s, f_{n}(s)\right) \in V_{n}$ and so $\rho\left(s, f_{n}(s)\right)<1 / n$.

We now show that $\Phi$ is uniformly continuous by showing that for $n \geqq 2$ and $(x, y) \in V_{n}$ we have $\rho^{*}(\Phi(x), \Phi(y)) \leqq 1 / n$.

Case (i). $N(x)<\infty, N(y)<\infty$. Then it is impossible that $n$ is strictly between $N(x)$ and $N(y)$, for if $N(x)<n<N(y)$, then $y \in V_{n}(S)$ and $(x, y) \in V_{n}$, from which it follows that $x \in V_{n}(S)$, a contradiction to $N(x)<n$. If $n$ is not smaller than $N(x)$ and $N(y)$, then $(x, y) \in V_{n} \subseteq V_{N(x)} \cap V_{N(y)}$ and so $N(x)=N(y)$, and $\Phi(x)=\Phi(y)$. If $n$ is not larger than $N(x)$ and $N(y)$, then, for each $r \geqq 1,\left(f_{r}(x), f_{r}(y)\right)$ $=\left(s_{i(N(x), x)}, x\right) \circ(x, y) \circ\left(y, s_{i(N(y), y)}\right) \in V_{N(x)} \circ V_{n} \circ V_{N(x)} \subseteq V_{n}$ and so $\rho\left(f_{r}(x), f_{r}(y)\right)<1 / n$. From this it follows that $\rho^{*}(\Phi(x), \Phi(y)) \leqq 1 / n$.

Case (ii). $N(x)<\infty, N(y)=\infty$. Then $x \in V_{n}(S)$ since $(x, y) \in V_{n}$ and $y \in V_{n}(S)$. Therefore, $N(x) \geqq n$. Then for $m \geqq n$, $\left(y, f_{m}(y)\right)$ $\in V_{m} \subseteq V_{n},(x, y) \in V_{n}$, and $\left(x, f_{m}(x)\right) \in V_{N(x)} \subseteq V_{n}$ and so $\left(f_{m}(x)\right.$, $\left.f_{m}(y)\right) \in V n$. Consequently, $\rho\left(f_{m}(x), \quad f_{m}(y)\right)<1 / n$ and so $\rho^{*}(\Phi(x), \Phi(y)) \leqq 1 / n$.

Case (iii). $N(x)=N(y)=\infty$ : For $m \geqq n,\left(x, f_{m}(x)\right) \in V_{m},(x, y) \in V_{n}$, $\left(y, f_{m}(y)\right) \in V_{m}$, and so $\left(f_{m}(x), f_{m}(y)\right) \in V_{n}$. As before, $\rho^{*}(\Phi(x), \Phi(y))$ $\leqq 1 / n$.

THEOREM 2. Let $S$ be a subset of a non-Archimedean uniform space $X$ and let $\rho$ be a uniformly continuous pseudo-ultrametric on $S$. Then there is a uniformly continuous pseudo-ultrametric $d$ on $X$ which extends $\rho$.

Moreover, if $S$ is either totally bounded or separable for $\rho$, then $d$ can be chosen so that $X$ has the same property for $d$.

Proof. Using the notation of Theorem 1, define $d$ on $X \times X$ by $d(x, y)=\rho^{*}(\Phi(x), \Phi(y))$. Since $\Phi$ is uniformly continuous, so is $d$.

If $S$ is totally bounded for $\rho$, then for each $n \geqq 2, I_{n}$ is finite and $\left\{s_{i} \mid i \in I_{n}\right\}$ is $(1 / n)$-dense in $S$ for $\rho$. From the definition of $\Phi$ it is easy to see that $\left\{s_{i} \mid i \in I_{n}\right\}$ is also $(1 / n)$-dense in $X$ for $d$. If $S$ is separable for $\rho$, then each $I_{n}$ is countable and $\bigcup_{n=1}^{\infty}\left\{s_{i} \mid i \in I_{n}\right\}$ is dense in $S$ for $\rho$ and therefore also in $X$ for $d$.

THeOREM 3. Let $S$ be a subspace of a non-Archimedean uniform space $X$ and let $\rho$ be a uniformly continuous pseudo-ultrametric on $S$ making $S$ complete. Then there is a function $f: X \rightarrow S$ which is uniformly continuous (for the given uniform structure on $X$ and the uniform structure on $S$ defined by $\rho$ ) and extends the identity function on $S$. 
Proof. In this case $\phi: S \rightarrow S^{*}$ is surjective. Define $f: X \rightarrow S$ by choosing for each $x \in X$ an element $f(x) \in \phi^{-1}(\Phi(x))$ with $f(s)=s$ if $s \in S$. Then for all $x, y \in X$,

$$
\rho(f(x), f(y))=\rho^{*}(\Phi(x), \Phi(y))=d(x, y) .
$$

Since $d$ is uniformly continuous, so is $f$.

COROLlaRy 1. Every complete subset of a pseudo-ultrametric space $X$ is a uniform retract of $X$.

COROLlaRY 2. Every uniformly continuous function from a complete subspace of a pseudo-ultrametric space $X$ into a uniform space has a uniformly continuous extension to $X$.

COROLlARY 3. Every uniformly continuous function from a subspace of a pseudo-ultrametric space $X$ into a complete Hausdorff uniform space has a uniformly continuous extension to $X$.

Proof. This follows immediately from Corollary 2 and [3, Theorem 2 , p. 190] by first extending to the completion of $S$, then extending to the completion of $X$, and finally restricting to $X{ }^{2}$

\section{REFERENCES}

1. R. A. Alo and H. L. Shapiro, Extensions of totally bounded pseudometrics, Proc. Amer. Math. Soc. 19 (1968), 877-884. MR 38 \#667.

2. R. F. Arens, Extension of coverings, of pseudometrics, and of linear-space-valued mappings, Canad. J. Math. 5 (1953), 211-215. MR 14, 1108.

3. N. Bourbaki, Elements of mathematics. General topology. Part I, Hermann, Paris; Addison-Wesley, Reading, Mass., 1966. MR 34 \#5044a.

4. R. Ellis, Extending continuous functions on zero-dimensional spaces, Math. Ann. 186 (1970), 114-122.

5. T. E. Gantner, Extensions of uniformly continuous pseudometrics, Trans. Amer. Math. Soc. 132 (1968), 147-157. MR 36 \#5886.

6. J. R. Isbell, On finite-dimensional uniform spaces, Pacific J. Math. 9 (1959), 107-121. MR $21 \# 4407$.

7. A. F. Monna, Remarques sur les métriques non-archimédiennes. I, Nederl. Akad. Wetensch. Proc. 53, 470-481 =Indag. Math. 12 (1950), 122-133. MR 12, 41.

University of Maryland, College Park, Maryland 20742

${ }^{2}$ The author is grateful to the referee for his suggestions. 